\title{
A Response to Andrea R. Halpern's Commentary
}

\author{
FREYA BAILES \\ Sonic Communications Research Group, \\ University of Canberra, Australia
}

\begin{abstract}
The author responds to points raised in Andrea Halpern's commentary, which appeared in Vol. 2, No. 1 of Empirical Musicology Review. Discussion focuses on the apparent contradiction between self-reports of veridical mental imagery of musical timbre, and cognitive constraints on temporal memory for multidimensional sound.
\end{abstract}

Submitted 2007 April 2; accepted 2007 April 6.

KEYWORDS: timbre, musical imagery, perception, memory, encoding

THE commentary by Andrea R. Halpern on "Timbre as an Elusive Component of Imagery for Music" (Halpern, 2007) is an importantly public contribution to discussion of the peculiarities of mental imagery for timbre. Professor Halpern is quite right to argue that imaging extended patterns of timbre may represent a challenging binding task for experiment participants. Based on her own experience and observations, she suggests that musicians might be better able to image timbre than 'non-musicians', linking her suggestion to a wider argument for individual differences in imagery ability. My intuitions in designing the experiments I report (Bailes, 2007) were indeed that imaging timbre, or rather using auditory imagery to facilitate performance in a perceptual task, would be a challenge. Consequently I invited only musicians to participate, defining 'musician' as at least undergraduate music student level at a UK university; a much stricter criterion than that used by Halpern of a minimum of two years of musical training. That these musicians struggled to image timbre is a finding I was anxious to share with the wider research community. As a performing musician myself, and having conducted many interviews with professional performers, conductors, and composers (e.g. Bailes, 2002), rich and veridical mental imagery of timbre is commonly reported. A principal motivation for research in this area must be to account for the apparent gulf between self-reports of complex imagery for timbre, and clear difficulties in the intentional imaging of music in laboratory experiments.

In my paper I tentatively speculate as to why imaging timbre might be different to imaging pitch and rhythm, and it seems that my speculation generally fits with Halpern's ideas. However, where I differ in my approach to timbre imagery is to view it as a primarily musical phenomenon. As Halpern writes, most research in this area concerns the imaging of an isolated instrument timbre (Crowder, 1989; Halpern, Zatorre, Bouffard, and Johnson, 2004; Pitt and Crowder, 1992). The important strength of this research is the controlled establishment of imagery properties such as the mean time required to report imaging an instrument tone (Crowder, 1989), spectral over dynamic features (Pitt and Crowder, 1992), and overlaps between imagery and perception of timbre in brain activation (Halpern et al., 2004). Such research focuses on the limitations of both short and long-term musical memory, with respect to encoding, retrieval, and rehearsal. Another approach is to consider the everyday experience of imaging music (Bailes, 2006), and to examine timbre as integral to music. This is not to ignore the cognitive limitations of memory for timbre, but rather to suggest that there is something special about memory for music that facilitates the temporal binding discussed in Halpern's commentary. As she has already observed (Warker and Halpern, 2005), depth of processing and the notion that timbre is in some way more essential to music than to speech might render music a special case. One challenge for researchers in this area is to 'tune into' the mental imagery of those with no particular musical expertise, but who, I venture to suggest, nevertheless image music from their favorite album with highly veridical timbre. Methodical investigations of timbre in imagery for music and not just isolated sounds are a valuable addition to our knowledge of auditory imagery.

Halpern discusses the interdependence of timbre and pitch, introducing experimental evidence that sometimes demonstrates, though at other times suggests minimal interference between the two sound 
dimensions. Again, she posits a role for individual differences in the ability or otherwise to dissociate pitch from timbre in imagery tasks. My discussions with experiment participants with absolute pitch also point to the interdependence of timbre and pitch in imagery, albeit at an anecdotal level. One who comes to mind is a pianist who was distracted in the pilot experiment by her uncharacteristic difficulties in imaging the pitches of the timbre sequence she had been asked to learn. She reflected that her absolute pitch was thrown by the use of sampled/digital sounds, and an inability to image the pitches accurately disturbed her conscious mental image for the timbre of the instrument pairs. In an experiment to be reported (Bailes and Delbé, 2007), I found differences in the magnitude of a priming effect when participants were asked to judge the intonation of a sung syllable, depending on whether the syllable was /di/ or /du/. While pitch and timbre are conceptually separable, they are ultimately related perceptions of one sound. The extent to which imagery for music resembles this perceptual relationship, yet varies with respect to individual differences, is an important question for its relevance to sound cognition more broadly.

I would like to respond to Halpern's critique of the experimental component of my paper. For clarity, I must point to the fact that my analyses of response time are for correct trials only, in both the pilot and the main experiment (see p. 25 and p. 30 of Bailes, 2007). Halpern writes that the data of interest from my studies are necessarily the comparison of imagery with control conditions. While I agree that this is the primary focus of the work, a comparison of these data with data from the perception task is not trivial. Given the paucity of research looking at extended musical imagery, particularly for timbre, we simply do not know, though we often assume, that perceiving the context in a discrimination task elicits superior response compared to imaging the context. Halpern herself compares data between imagery and perception tasks when she provides comparative statistics from her own work (Halpern et al., 2004; Zatorre and Halpern, 1993) and research by Crowder (1989).

One of the difficulties of research in mental imagery is the selection of an appropriate control task. The experiments I report are no exception, and the findings would be more convincing still with additional control tasks. Halpern proposes a control in which participants first learn the stimulus music, and then perform a control task identical to the imagery task except that no instructions to image the music are provided. The difficulty with her proposed method is that participants having learnt a piece of music are likely to spontaneously image its continuation when it is interrupted, even without explicit instruction to do so. For a true control it would be necessary to somehow prevent the accidental imaging of the music. In the unreported study mentioned above (Bailes and Delbé, 2007), already familiar nursery rhyme melodies are used as stimuli. The first few notes are presented, and then participants are required to image the melody continuation. As a control condition, participants are informed that the opening notes belong to a familiar theme of classical music, beginning with the same combination of pitches. We found that participants who are more familiar with the nursery rhyme melodies than the classical themes inadvertently image the nursery melodies, even though no reference to the nursery melodies was made, a few notes only were presented, and participants were instructed to image something quite different. In Bailes (2007), I chose instead to ask participants to make timbre judgments before knowing the stimulus music to avoid contaminating the control task with an uninvited imaging of the piece. Halpern appropriately wonders whether simply learning the music was sufficient to facilitate performance on the imagery task compared to the control task. However, as I argue in the paper, improved performance merely due to repeated listening should be discernible as a practice effect across trials. No such effect was found. Consequently, I believe we do "have evidence from a new kind of indirect test of the ability of people to imagine more complex timbres than have been used previously" (Halpern, 2007, p. 36).

The new method I employ in Bailes (2007) is based on inference, and cannot directly measure the temporal extent of the imagery experienced by participants. Moreover, as my paper openly argues, it seems that while experiment participants accurately represented the timbre of the music, they were not necessarily able to create a mental image. Halpern reflects on whether experiment participants might have encoded the stimulus timbre in abstract or verbal form, though it is difficult to understand how participants could verbally encode the relative filtering used in stimuli from the main experiment. I concur with definitions in the literature of mental imagery as a conscious re-presentation sharing the qualia of sensory experience. A specific aim in publishing this research has been to highlight difficulties in creating such imagery for timbre under laboratory conditions. Halpern mentions her own 'bottom drawer' experiments in which she failed under certain circumstances to demonstrate memory for timbre. Perhaps we should view such difficulties as a finding worthy of discussion in its own right, as a number of research questions ensue. How is it, for example, that such a mismatch occurs between the reported difficulty of generating the timbre of a 
piece of music learned in an experiment, and the everyday experience of imaging the full timbre range of a favorite track?

Finally, Halpern interprets the finding by Pitt and Crowder (1992) that participants are better able to image the spectral component of timbre than the dynamic component as a possible explanation for the apparent difficulty of imaging timbre through time. This is an intriguing hypothesis, projecting an effect that occurs at the equivalent of a micro level of perception to the macro structure of a note sequence. Certainly, limitations of working memory would be expected to shape any individual's ability to image the timbre of extended musical passages. I suggest that, in order to unravel the apparent contradiction between difficulties of imaging timbre and self-reports of veridical timbre imagery for favorite and frequently heard or performed music, we should examine the encoding of timbre at perception. After all, timbre plays a role in conveying important information to a listener (e.g. nature of the sound-producing event), while its role in memory is less apparent. Level of processing (see Warker and Halpern, 2005), and strong affective response to music, in addition to sheer repetition, might facilitate the encoding in memory of sounds which are 'meaningful' to the individual.

\section{REFERENCES}

Bailes, F. A. (2002). Musical Imagery: Hearing and Imagining Music. PhD thesis, University of Sheffield.

Bailes, F. A. (2006). The use of experience-sampling methods to monitor musical imagery in everyday life. Musicae Scientiae, Vol. 10, No. 2, pp. 173-190.

Bailes, F. A. (2007). Timbre as an elusive component of imagery for music. Empirical Musicology Review, Vol. 2, No. 1, pp. 21-34.

Bailes, F., \& Delbé, C. (2007). Long-term Melodic Expectation: The Unexpected Observation of Distant Priming Effects. Manuscript in progress.

Crowder, R. G. (1989). Imagery for musical timbre. Journal of Experimental Psychology: Human Perception and Performance, Vol. 15, pp. 472-478.

Halpern, A. R. (2007). Commentary on "Timbre as an elusive component of imagery for music," by Freya Bailes. Empirical Musicology Review, Vol. 2, No. 1, pp. 35-37.

Halpern, A. R., Zatorre, R. J., Bouffard, M., \& Johnson, J. (2004). Behavioral and neural correlates of perceived and imagined musical timbre. Neuropsychologia, Vol. 42, pp. 1281-1292.

Pitt, M. A., \& Crowder, R. G. (1992). The role of spectral and dynamic cues in imagery for musical timbre. Journal of Experimental Psychology: Human Perception and Performance, Vol. 7, pp. 28-38.

Warker, J. A., \& Halpern, A. R. (2005). Musical stem completion: Humming that note. American Journal of Psychology, Vol. 118, pp. 567-585.

Zatorre, R. J., \& Halpern, A. R. (1993). Effect of unilateral temporal-lobe excision on perception and imagery of songs. Neuropsychologia, Vol. 31, pp. 221-232. 